Revue musicale OICRM

\title{
Instrumentaliser la subjectivité de la perception au sein d'un paysage sonore interactif. L'installation interactive et immersive Soleil
}

Jean-Ambroise Vesac

Volume 4, numéro 1, 2017

URI : https://id.erudit.org/iderudit/1040301ar

DOI : https://doi.org/10.7202/1040301ar

Aller au sommaire du numéro

Éditeur(s)

Observatoire interdisciplinaire de création et recherche en musique (OICRM)

ISSN

2368-7061 (numérique)

Découvrir la revue

Citer cet article

Vesac, J.-A. (2017). Instrumentaliser la subjectivité de la perception au sein d'un paysage sonore interactif. L'installation interactive et immersive Soleil. Revue musicale OICRM, 4(1), 86-107. https://doi.org/10.7202/1040301ar
Résumé de l'article

Cet essai discute la composition musicale liée au geste ayant fondé le dispositif d'interaction Soleil. Cette oeuvre demande à l'expérimentateur de prendre conscience de son comportement, afin de lui donner la possibilité de l'associer à un environnement sensoriel, par la transposition musicale de ses gestes et l'adaptation rythmique du dispositif à ses réactions physiologiques. 


\title{
Instrumentaliser la subjectivité de la perception au sein d'un paysage sonore interactif. L'installation interactive et immersive Soleil
}

\author{
Jean-Ambroise Vesac
}

\section{Résumé}

Cet essai discute la composition musicale liée au geste ayant fondé le dispositif d'interaction Soleil. Cette œuvre demande à l'expérimentateur de prendre conscience de son comportement, afin de lui donner la possibilité de l'associer à un environnement sensoriel, par la transposition musicale de ses gestes et l'adaptation rythmique du dispositif à ses réactions physiologiques.

Mots clés : art numérique; dispositif d'interaction sonore ; immersion; spatialisation.

\section{Abstract}

This essay discusses the musical composition linked to a gesture of the head. This interaction design founded the project Soleil(Sun). This artwork asks the experimenter to be aware of his behaviour to get associated with a sensory environment. His physiological reactions are transposed to rhythmics of light and sounds.

Keywords: digital art; immersion; sound interaction device; spatialization. 


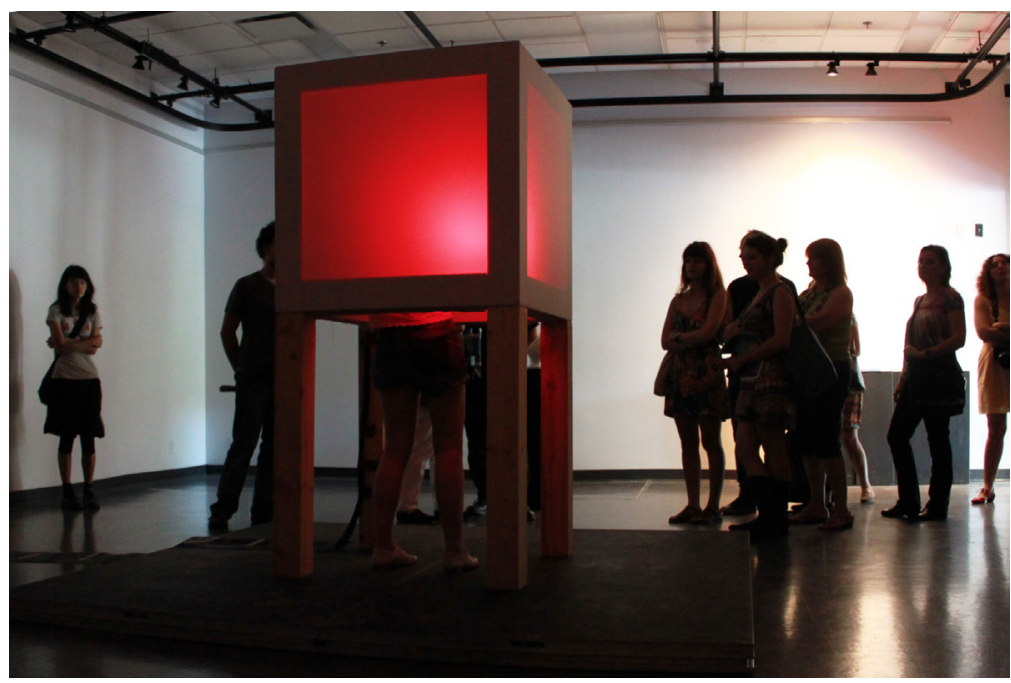

Figure 1 : Vue d'ensemble de l'installation Soleil, Biennale internationale d'art numérique de Montréal, 2014. Photo : JAV.

\section{Présentation De L'installation}

Cet essai présente l'expérience de composition musicale liée au geste ayant fondé l'œuvre Soleil et son projet de médiation culturelle, dans leur version présentée dans le cadre de la Biennale d'art numérique de Montréal à la Maison de la culture de Montréal-Nord, au printemps 2014. Soleil ${ }^{1}$ est une installation interactive et immersive dans laquelle le visiteur expérimente des stimulations sensorielles (paysages sonores et couleurs) qui jouent avec sa perception de l'espace pour le transporter dans une étendue imaginaire. Soleil demande à l'expérimentateur de prendre conscience de son comportement, afin de lui donner la possibilité de l'associer à un environnement sensoriel, par la transposition musicale de ses gestes et l'adaptation rythmique du dispositif à ses réactions physiologiques.

Dans l'installation, les flashs lumineux et sonores, hypnotiques et centrifuges, structurent le temps de la perception par la réponse physiologique du système oculaire du participant. Par cet effet, la lumière organise une alternance d'effets d'immersion créant une mise à distance, critique, ainsi qu'une immersion incorporante. En réaction à ces changements, l'activité cognitive du participant s'exprime par un changement de posture corporelle. Le système analyse alors l'extériorisation de cette réaction intérieure. La dimension sonore accompagne la dimension visuelle, dans ses effets immersifs, pulsatifs et spatiaux. La composition liée des dimensions sonores et visuelles amène le participant à ressentir un effet de synchrèse, défini par Michel Chion comme

un phénomène psycho-physiologique spontané et réflexe, dépendant de nos connexions nerveuses et musculaires, et qui consiste à percevoir comme 
un seul et même phénomène se manifestant à la fois visuellement et acoustiquement la concomitance d'un événement sonore ponctuel et d'un événement visuel ponctuel, dès l'instant où ils se produisent simultanément (Chion 2010, p. 164).

Ces effets d'immersion « dissolvent la distinction entre soi et l'environnement et créent une mixture corps/monde » (Jones 2006, p. 15).

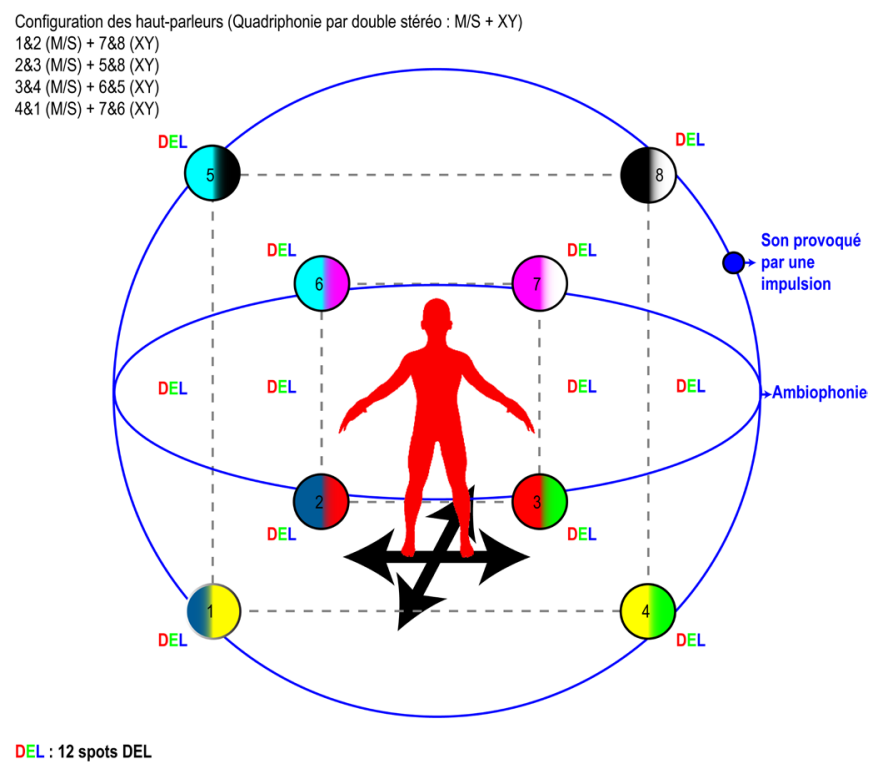

Figure 2: Disposition des haut-parleurs et des canaux de diffusion sonore.

Du point de vue sonore, l'expérimentateur est immergé dans un univers sonore 3D. La composition est une suite de parcours dans des paysages sonores urbains. Différentes ambiances sonores sont traversées, par exemple des parcs de jeux, des ruelles, des escaliers, autant de lieux choisis pour leur résonnance acoustique et sociale. La composition est ponctuée de points d'écoute s'entremêlant et dont le participant découvre la musicalité par son interaction corporelle. Le son provient de huit haut-parleurs disposés tout autour de lui à l'intérieur d'un cube (figure 2). Le son dans l'installation est utilisé pour suggérer à l'expérimentateur qu'il se déplace à travers différents lieux. Ces lieux sont identifiables à leur réverbération acoustique qui trahit leur volume. Le sens de l'orientation de l'expérimentateur est sollicité par des déplacements des masses sonores, du timbre et de la réverbération acoustique, en association aux effets de lumière. 
L'intention initiale ${ }^{2}$ était de réaliser un dispositif ${ }^{3}$ audiovisuel interactif permettant de procurer une expérience médiatique immédiate. L'interaction est basée sur des gestes simples, sans recours à la navigation hiérarchique (menu). L'œuvre est plutôt ludique et "grand public ». Cette finalité axée sur "l'attention portée au corps du spectateur » (Alberganti 2013, p. 8) associe ce dispositif à l'art de l'installation.

Cependant, les dispositifs impliquant une interaction gestuelle simple n'ont, la plupart du temps, que peu de déploiement tempore ${ }^{4}$. Ils sont comme des miroirs déformants traduisant les mouvements en temps réel. La perte rapide d'intérêt de ces propositions provient de "l'insatisfaisant modèle d'une communication symétrique » (Ungeheuer 2013, p. 1377) et leur trop grande prédictibilité. Dès lors que la métaphore d'interaction est comprise - ce qui se produit assez vite -, la curiosité s'épuise. En conséquence, comment développer une interaction corporelle simple qui résolve la contradiction apparente entre son évolution (non prédictible) et son accessibilité (aisée) ?

\section{Agentivité ET SUBJeCtivité}

Afin d'enrichir l'expérience d'interaction, une première piste est proposée. Le dispositif attire l'attention du participant, et surtout la retient, par la mise en évidence de son agentivité (agency) ${ }^{5}$ dans l'œuvre. Le participant est à même de constater les conséquences de ses actions dans le contenu même. Ce type de médiation a pour effet d'engager la volonté du participant (Falcão 2012) et de permettre le dépassement du niveau de l'interaction opérationnelle par le couplage dynamique du participant à l'œuvre (Hansen 2004). Par ses choix successifs, le participant est en quelque sorte

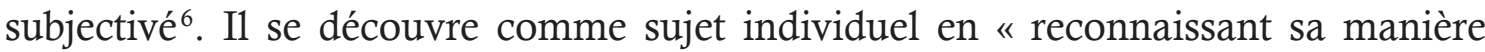

2 En 2006, Marcin Sobieszczanski, professeur-chercheur à l'Université Nice Sophia Antipolis, nous demandait de lui proposer un projet sur le thème du biofeedback en art contemporain.

3 " Un dispositif indique un arrangement qui a un effet normatif sur son "environnement" puisqu'il y introduit certaines dispositions [immédiates pour l'activité qui sera réalisée] " (Raffnsøe 2008, p. 47). «Un dispositif est tout ce qui a, d'une manière ou d'une autre, la capacité de capturer, d'orienter, de déterminer, d'intercepter, de modeler, de contrôler et d'assurer les gestes, les conduites, les opinions et les discours des êtres vivants » (Agamben 2007, p.8).

4 Donnons un exemple : l'installation Notional Field (2012) des artistes Annica Cuppetelli et Cristobal Mendoza présenté au Mois Multi (Québec) en février 2014 donne un exemple d'une interaction symétrique, où «les déplacements [du spectateur] [... ] viennent activer le mouvement des lignes lumineuses ». Voir le programme en ligne : http://mmrectoverso.org/wp-content/uploads/2016/01/ProgrammeMM 2014.pdf (p. 25); et également : http://cuppetellimendoza.com (consultés le 6 juin 2016).

5 La définition de la notion d'agence utilisée dans cette recherche fait appel à la faculté d'émergence et d'inspiration à l'action d'un objet sur un sujet, telle que définie par Alfred Gell (2009) dans sa théorie anthropologique des relations sociales entourant les œuvres d'art (art agency). Selon Gell, l'œuvre d'art agit comme un agent accomplissant une influence sur des patients, les destinataires de l'œuvre. Suivant cette perspective, l'œuvre d'art est réputée capable de motiver à l'action dans le réel. En complément, la notion d'agence est pour Pickering (1995) un phénomène d'émergence, de dépassement des conditions initiales provoqué par une relation expérimentale de recherche entre des agents humains et non humains.

6 "L'homme comme sujet [...] sub-jectum, le "sous-jacent" sur la base duquel tout doit désormais reposer »(Renaut 1988, p. 57). 
de donner sens au réel, au moyen de son activité de symbolisation " (Richard et Wainrib 2006, p. 2).

De manière générale, le corps est au sein de l'interface qui l'instrumentalise. Il participe au dispositif qui est pour lui une sorte d'extension. Cette prolongation numérique du corps démultiplie son action potentielle, notamment dans l'œuvre. Malgré cela, obtenir des données permettant de cerner l'expérience subjective à partir d'une observation physiologique est extrêmement délicat. Une partie de la difficulté réside dans la formalisation des données biologiques du fait de la grande différence entre l'émotion ressentie et l'activité physiologique l'accompagnant, dans sa normalité comme dans ses extrêmes. En plus d'être parfaitement individuel, le ressenti de l'activité intérieure - des organes comme le cœur - en réaction à des situations est indissociable d'une perception de soi produite par l'expérience.

Pour l'observation du corps, de nombreuses technologies, intrusives ou non, permettent de prendre une mesure quantitative de son activité. L'une des plus utilisées, pour sa simplicité d'usage, est la conductivité épidermique qui permet la mesure du stress. En situation de stress, le corps se durcit et réduit ainsi sa conductivité électrique. Mesurées sur le bout du doigt, les variations de tension nous informeraient de la nervosité ${ }^{7} \mathrm{du}$ participant. Cette mesure s'apparente à un axe allant d'une valeur minimale à une valeur maximale, ce qui correspond à la résistance progressive et linéaire d'un potentiomètre.

Il existe de nombreux outillages de mesure physiologique, mais la difficulté « de faire du sens " reste entière, car peut-on véritablement induire le sens d'une réaction physiologique ? Dans le contexte d'une production artistique, l'accès à des outils d'analyse adéquats n'est pas facile. Il y a des limites à utiliser le « corps vivant », ses signaux biologiques, pour tenter de cerner l'expérience subjective de l'agentivité du dispositif sur le participant et s'en servir pour le développement de l'interactivité. Est-il possible d'apprécier, un peu plus justement, l'expérience subjective du participant par sa " corporalité vivante", par les mouvements qui en sont issus ? Autrement dit, est-il possible d'instrumentaliser la subjectivité de la perception elle-même, dans les traces qu'elle imprime aux mouvements du corps ? L'hypothèse est que l'instrumentalisation des mouvements (perception-action) fournit des données sur le participant, sur sa perception du dispositif, permettant de caractériser son comportement. Cette caractérisation opère au cœur du programme informatique comme un miroir comportemental. Face à un avatar de lui-même, le participant discerne son agentivité dans les variations audiovisuelles, dans les variations sémantiques de contenu et de tempo.

Considérons la perception comme irréductible au sujet et constitutive de sa subjectivité. C'est l'expression d'une "intentionnalité incarnée ", pour paraphraser Merleau-Ponty pour qui le corps est "le moi naturel du sujet de la perception " (Carman 1999, p. 224). Dans la perception d'un phénomène extérieur, il y a toujours

$7 \quad$ Un exemple de l'usage de cette technologie est l'œuvre de Lynn Hugues et Simon Laroche, Perversely Interactive System (2004). Voir : www.interstices.uqam.ca/fr/realisations/lynn-hughes/item/27-perversely-interactive-system.html (consulté le 6 juin 2016). 
un peu d'autoperception. Maintenant, quel est l'intérêt de ce chiasme perceptif pour cette recherche-création ${ }^{8}$ ?

\section{Perception-Action ET INSTRUMENTALISATION}

Le paradigme central est le lien entre perception et action, qui est confirmé par la théorie motrice de la perception qui « envisage l'existence de processus commun à la perception et à l'action " (Nicolas et Doré-Mazars 2002, p. 26). Dans le but d'enrichir l'expérience d'interaction, nous cherchons un mode de perception, une "écoute intelligente" (Chion 2010, p. 254) qui a le potentiel de convoquer "des mécanismes d'ordre supérieur ou processus cognitif » (Botte et al. 1989, p. 141), l'imagination par exemple. Il est donc établi que nos perceptions agissent sur nos actions, car « notre rapport au monde est changé par les informations que nous percevons, qui sont la matière avec laquelle nous fabriquons nos opinions et nos pensées les plus riches et les plus diversifiées. » (Chion 2010, p. 255). L'intérêt du couple perception-action est déterminant dans les formes d'art développant de nouveaux médiums d'expression, notamment les arts numériques.

Par conséquent, comment instrumentaliser, formaliser la subjectivité de la perception ? Selon Roger Reynolds, l'instrumentalisation du corps par lui-même " impose un certain nombre de contraintes et détermine en conséquence un champ spécifique de possible " (dans Bioteau 2013, p. 1345). Dans le champ des possibles $\mathrm{du}$ corps, la tête centralise l'écoute et le regard, du moins dans leur condition naturelle. Ainsi, par l'instrumentalisation de l'orientation de la tête, c'est l'écoute, le regard et peut-être davantage qui sont instrumentalisés, captés. Le regard devient un instrument de contrôle dans l'interface qui a formalisé ses mouvements dans des catégories déterminées - par l'artiste dans ce cas-ci. Les mouvements de la tête sont nécessaires à l'orientation de la perception et à la perception de l'orientation. En effet, l'audition a une triple fonction de perception sonore, d'orientation spatiale et de centre de l'équilibre. La spatialisation est importante dans l'articulation musicale, parce que, toujours selon Reynolds, "l'espace est une dimension immanente de la musique " (ibid., p. 1337). En conséquence, ni le corps ni la musique ne peuvent être séparés de leur espace d'action. L'espace d'action du corps est inscrit dans son schéma corporel. Pour le son, l'espace d'action est le milieu informationnel (Piché 2010, p. 57), la réverbération et l'ambiance de «l'espace entourant sa production» (ibid., p. 11) : «Par l'audition, nous percevons des informations sur l'évènement sonore initial ainsi que sur son contexte environnemental » (ibid., p. 66). Ce point sera repris.

Nous savons donc que nous allons appareiller la tête du participant (figure 3). Les lunettes sont une instrumentalisation de la vue qui, par l'effet de leur affordance, nous demande de voir. Car, déplacer la tête pour observer mieux, c'est le résultat d'un choix, c'est la décision consciente de se tourner vers quelque chose dont la vue nous

8 « [...] un projet de recherche-création engendre deux types de résultats. Il y a, d'une part, la création elle-même et, d'autre part, la réflexion théorique scientifique qui accompagne la création et qui vise à communiquer rigoureusement le processus de création lié à l'œuvre créée » (Dubé 2013, p. xiii). 
interpelle. Ainsi, il suffit d'un senseur gyroscopique ${ }^{9}$ sans fil sur une paire de lunettes posée sur le nez du participant pour capter et transmettre l'orientation de la tête, et donc de l'orientation de la vue et de l'ouie.

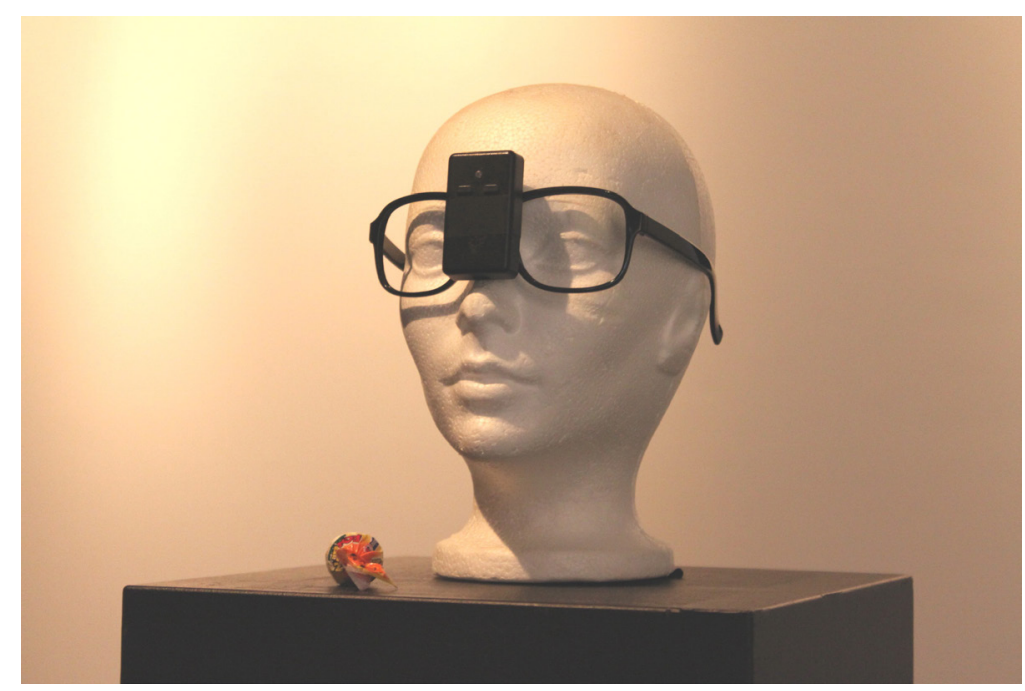

Figure 3 : Paire de lunettes interactive. Photo : JAV.

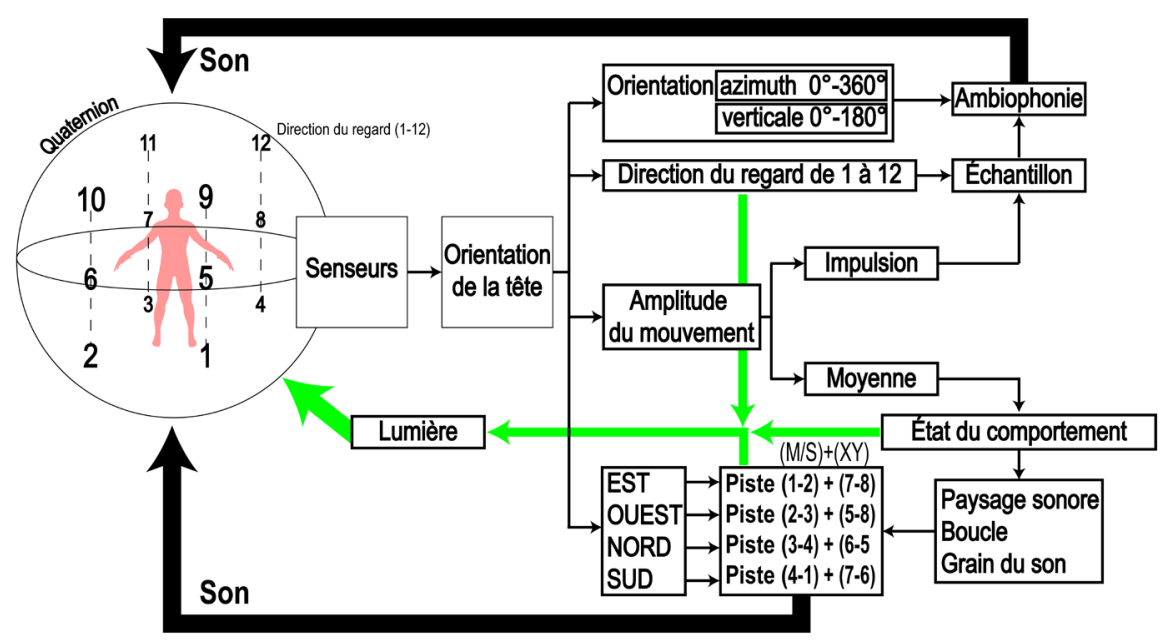

Figure 4 : Schéma fonctionnel de l'installation Soleil.

Quelles sont les perspectives? Par l'instrumentalisation de la tête du participant, nous allons en même temps recueillir, sans pouvoir les différencier, des réactions involontaires ou utilitaires à l'orientation spatiale et des réactions à l'écoute, ce qui pourrait nous informer sur ces préférences dans l'hypothèse où la tête se tournerait vers ce qu'elle aime le mieux, pour mieux le percevoir. 
Or, la capacité de percevoir la musique et la spatialisation sonore dépend, au moins en partie, de la capacité du corps à posséder une "mémoire physique » (embodiement) du geste musical, la perception étant dépendante de la capacité d'agir ${ }^{10}$ (Schellenberg 2011). Ainsi, la relation perception-action est confirmée par Neisser :

There is a two-way process here where sound induces images of movement, and conversely, where previously learned images of sound-related movement are projected onto sound, actually manifesting an instance of what has been called the perceptionaction cycle in the cognitive sciences (dans Godøy 2009, p. 106).

\section{RECONNAISSANCE DE SOI DANS L'ACTION}

Pour que les conditions soient favorables au développement de l'effet d'agentivité, le sujet doit être conscient de lui-même et de ces actes, d'autant plus que la reconnaissance de son action, la réactivité, est le premier degré nécessaire à l'expérience d'interaction. La réactivité symétrique du dispositif est la confirmation primordiale de son "fonctionnement ». La reconnaissance de nos actions peut se retrouver à plusieurs niveaux perceptifs. Pour en rendre compte, nous adaptons ici la classification de Fourneret (2002, p. 260) qui détermine trois stades de conscience de soi dans l'action soit : 1) «la représentation motrice de l'action » (le geste automatique, physiologique, non conscient), 2) "l'émergence consciente de certains aspects du contenu de la représentation motrice en action » (le geste "instrumental »), et enfin 3) le sens de l'agentivité « une rétroaction de haut niveau faisant référence au contenu sémantique de l'action » (le geste de commande).

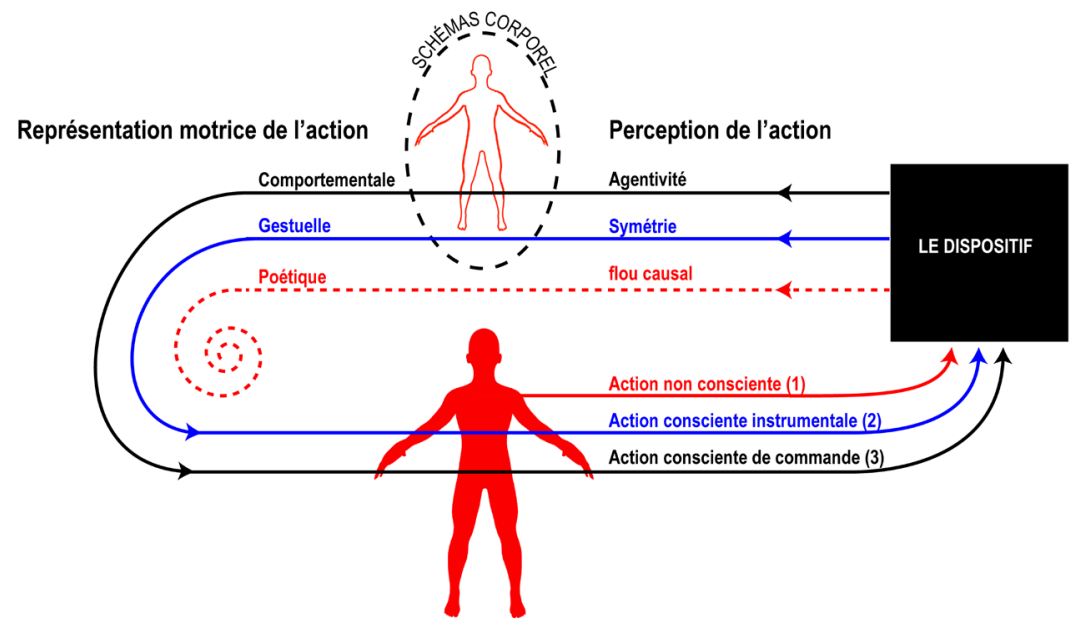

Figure 5 : Niveaux de reconnaissance de soi dans l'action.

10 Selon Schellenberg (2011), notre capacité à percevoir un objet en 3D résulte de notre mémoire prédictive du mouvement autour de l'objet. Par ce détachement perceptif, notre cognition est en mesure de «faire le tour d'un objet à distance » et ainsi à le percevoir dans l'espace. 
Le premier stade de la reconnaissance de soi dans l'action ${ }^{11}$ est non conscient. C'est le réflexe du geste physiologique. Certains réflexes physiologiques marquent un état d'écoute active, concentrée et focalisée. Succédant à un son vif et puissant, un mouvement de la tête involontaire (startle response), est une réaction de surprise provoquant une attention accrue (Farnell 2010, p. 108). Un autre réflexe nous paraît intéressant au regard de l'interactivité sonore proprioceptive. "L'orientation réponse » est un mouvement involontaire de la tête qui tourne sur elle-même pour améliorer, résoudre la localisation d'un son vif (ibid.). En effet, cette rotation permet de percevoir l'origine du son par la différence de phase, l'écart temporel entre les deux oreilles. Ainsi, un « accident» dans la composition paysagère provoque une réaction du participant, un mouvement, qui est traduit comme un "geste " provoqué par le choc entre son imaginaire et l'espace sonore. Ce sont aussi les gestes de toutes sortes qui accompagnent l'écoute musicale sans produire de bruit (Godøy 2009, p. 110), comme hocher de la tête. Ils marquent une rupture facilement détectable informatiquement par l'amplitude du mouvement. Ils présentent l'intérêt d'être répétitifs et d'établir des rythmes. D'autre part, l'occultation de l'évidence causale, le « flou causal », ouvre à l'interprétation poétique.

Le second stade de reconnaissance de soi dans l'action est le geste musical direct. C'est un geste de contrôle de la production/synthèse sonore, comme celui que l'on applique sur un instrument de musique. Le geste et le son ont une relation symétrique. Cette relation formalisée s'apparente à l'utilisation d'un instrument, comme pourrait le faire un interprète. Il est facilement reconnaissable par son rapport rétroactif, mais contrairement à un instrument de musique, dans un dispositif d'interaction sonore gestuelle, comme nous l'avons remarqué plus tôt, l'expression est limitée par la fixation de son caractère symétrique.

Le troisième stade de reconnaissance de soi dans l'action est l'agentivité du geste de commande. L'acte de commande est plus complexe à décrire que l'action directe de manipuler un bouton de contrôle. Il exige " de connaître l'état actuel, les facteurs déterminant dynamiquement sa situation et l'état visé (ou idéal) » (Ungeheuer 2013, p. 1378). L'acte de commande est rendu possible par la combinaison d'une «écoute engagée "- c'est-à-dire " un état supercritique d'expérience impliquant un certain degré d'interactivité, une compréhension et une implication complètes avec le son perçu et les processus de sa production ${ }^{12}{ }$ (Farnell 2010, p. 107) - et de l'expression d'une intention compositionnelle modifiant un ensemble de paramètres dépassant et englobant le niveau d'expression instrumentale propre au second stade de reconnaissance de soi dans l'action. Le corps est alors dans une relation de couplage où « le son provoque des réactions physiques qui à leur tour provoquent du son ${ }^{13}$ " (ibid.) et de nouvelles perspectives de développement. Dans le cas de la spatialisation sonore, cette

11 Ce sujet a été notamment étudié par Marc Jeannerod (2006).

12 "Engaged listening is a supercritical state of experience that involves some degree of interactivity, being complete understanding and involvement with the sound we are hearing and its causal process " (notre traduction).

13 "The sound may drive physical responses, while the responses also drive the sound (notre traduction). 
rétroaction de haut niveau offre au participant la liberté de jouer avec sa perception de l'espace et de s'y projeter. Comme nous l'avons vu, l'orientation et le comportement sont indissociables.

Dès lors, le contrôle del'orientation et la spatialisation del'attention dans le processus de commande musicale interactive impliquent de jouer avec son comportement. Le système est alors dans une boucle récursive comportementale. Nous pouvons alors parler d'agentivité. L'instrumentalisation de la perception sonore proprioceptive offre donc une issue nouvelle au développement de l'interaction gestuelle, par l'implication de l'agentivité dans les processus de contrôle.

En résumé, l'instrumentalisation de l'expérience subjective permet une mise en œuvre de l'agentivité dans les processus récursifs de contrôle audiovisuel de haut niveau, ce qui est un moyen de faire évoluer une installation d'interaction corporelle tout en conservant son accessibilité.

MATÉRIAUX SONORES

Revenons maintenant à l'œuvre Soleil. Où se situe notre démarche de création ? Elle prend, en partie, ses racines dans l'art radiophonique à cause de son "goût pour l'ubiquité » (Kaltenecker 2013, p. 509) qui s'exerce par le rapprochement et le montage, mais aussi par l'utilisation équilibrée du «bruit » dans le moment d'écoute musicale. Car, si le silence est l'origine de l'organisation de la musique, le traitement et l'usage du son-bruit ${ }^{14}$ est à l'origine de son esthétique ${ }^{15}$ (Lussac 2013, p. 545).

La composition musicale cherche un point d'équilibre de la tension dans l'écoute entre l'imprévisibilité et la continuité, qui se traduit par une variation de l'opacité de la masse sonore. Densité et complexité sonores sont partiellement allégées par le rappel sémantique d'une réaction sonore à un geste, peut-être accidentel, du fait de la correspondance à ce geste de la nature du moment où l'échantillon sonore a été enregistré, correspondant tous deux à un moment spontané. Cette sorte de sonification du geste du participant lui permet de retrouver la résonnance de son geste à l'intérieur de l'écriture sonore, au travers des couches entremêlées. L'observation de l'activité de médiation nous apprend que les ponctuations sonores sont discernées par l'effet de contraste qu'elle génère dans le paysage sonore, en référence à la situation sociale suggérée.

De manière concrète, la collecte des sons est effectuée principalement en extérieur (figure 6), selon une "écoute in situ qui se caractérise par une sélection réflexe ou consciente d'éléments pertinents et le refoulement des autres » (Chion 2010, p. 147). La captation n'est pas «transparente », mais consiste plutôt en un parcours sonore performé, apparenté dans sa démarche aux marches sonores de Hildegarde

14 «Il faut rompre à tout prix ce cercle restreint de sons purs et conquérir la variété infinie des sons-bruits » (Russolo 1975, p. 37).

15 «Le bruit comme fondement d'une autre esthétique sonore, naturelle, industrielle » (Lussac 2013, p. 545$)$. 
Westerkamp ${ }^{16}$, dans lesquelles « les choix séquentiels sont la signature artistique de l'œuvre » (Mariétan 2005, p. 32-33).

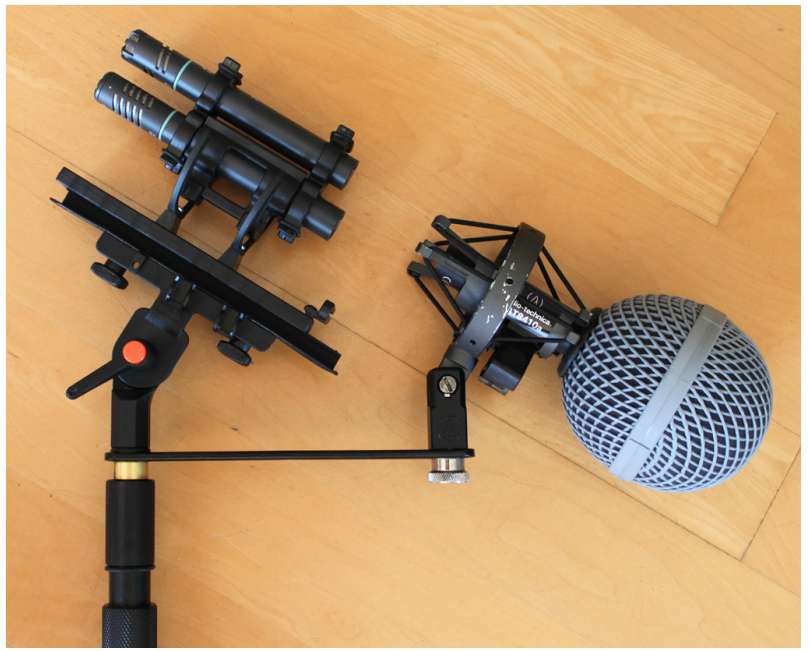

Figure 6 : Ensemble de microphones, à l'avant, une prise de son stéréo Mide/Side avec un AKG CK94 et CK91 ; à l'arrière, une prise en stéréo XY avec un Audio Technica BP4025. Photo : JAV.

L'utilisation de matériaux sonores issus d'enregistrement extérieur (field recording) se rapproche de la musique concrète, mais à la différence de l'école de Paris (Ina-GRM) pour qui le son devient un objet sonore (Schaeffer 1966) par sa distanciation de son contexte d'origine, notre approche conserve « clairement la nature du matériel ou de la réalité concrète qui lui a donné naissance ${ }^{17}$ " (Maciunas 1990, p. 146) et en cela tient du " concrétisme ", qui n'est pas éloigné de « la tradition de l'empirisme » de Morton Feldman et l'École de New York (Bosseur 2013, p. 629).

Le contexte de la prise de son dans l'enregistrement est nécessaire à la restitution de la spatialité. Il ajoute également un aspect social propre à l'évocation d'images, au souvenir. De l'axe temporel, « la vie ordinaire forme la matière privilégiée des nouvelles techniques d'enregistrement » (Piché 2010, p. 510), provoquant de nouvelles durées. Ces durées prises dans les déplacements du quotidien sont connues du corps qui les vit autrement que musicalement. Là pour nous réside une musicalité de tous les jours, qui parle de la vie, ce qui nous ramène à la pensée de Jankélévitch sur « la musique comme une expérience vécue à même la vie » (Jankélévitch 1983, p. 118).

Une installation offre la situation nécessaire au «moment de l'écoute " (Chion 2010, p. 254). Les conditions techniques sont rassemblées dans un lieu de l'art. Il reste au participant à s'engager, à se rendre disponible à l'expérience, à percevoir avec " ouverture ". Cette disposition curieuse devant un objet ou une situation artistique fait partie de notre proposition thématique. C'est donc ce « mouvement» vers l'œuvre

16 À propos d'Hildegard Westerkamp, voir : www.sfu.ca/ westerka (consulté le 6 juin 2016).

17 "A material or concrete sound is considered one that has close affinity to the sound producing material - thus a sound whose overtone pattern and the resultant polychromy clearly indicates the nature of material or concrete reality producing it » (notre traduction). 
que nous cherchons à instrumentaliser et ainsi l'utiliser dans l'œuvre même. En effet, si « écouter c'est agir!" (Chion 2010, p. 255), il ne reste qu'un pas vers l'interaction. Cette instrumentalisation transforme l'œuvre en un " espace d'interaction » où elle s'incarne, en paraphrasant Ligeti, comme un processus (Sabbe 2013, p. 1091) de commande (Ungeheuer 2013) au cœur de l'interaction électronique en direct. Notre installation propose une expérience « d'ergo-audition » dans laquelle l'auditeur " est en même temps, sous une forme ou sous une autre, l'émetteur du son entendu » (Chion 2013, p. 98).

\section{Programmation ET SUBJECTIVITÉ MACHINIQUE}

$\mathrm{Du}$ point de vue de la matière informatique, la programmation fait partie de la démarche de création. C'est une activité objective, où les données et les processus sont déterminés, les résultats aussi ${ }^{18}$. La programmation est rarement décrite comme une forme de création, mais plutôt l'opposé, " [1]es raisons invoquées étaient que l'on ne pouvait pas faire de l'art avec des machines déterministes qui ôtaient toute liberté aux artistes »(Couchot et Hillaire 2013, p. 10). Pourtant, dans les arts numériques, la "pensée opérante ", le calcul automatique et tous les dérivés produits par un ordinateur sont des moyens potentiels d'expression. Il y a cependant une difficulté fondamentale à surmonter : 1'art ne peut être uniquement formalisé, c'est contre sa nature même ; il doit y avoir du " flou artistique ", une indétermination (Cage et Tudor 1959). Alors, comment faire entrer de la poésie dans le programme? Au milieu de la formalisation la plus stricte et l'objectivité booléenne (vrai ou faux), il est possible d'encapsuler un agent humain et de prendre ses réactions (captées par des senseurs) comme un générateur de possibilités. Ainsi, sa subjectivité participe à l'édification du "moment de l'art ». En effet, quand le participant pose un geste, il génère une donnée objective (objectivité de l'action) et en retour le système réagit en produisant un son, un évènement vérifiable (il y a bel et bien, oui ou non, un son) objectivement. Dépassant la réaction, les intentions préalables à l'action du participant proviennent de sa subjectivité que nous qualifions de participante pour insister sur son implication. De l'autre côté, le processus de catégorisation, de formalisation des données est une expression de la subjectivité du concepteur du programme et devient en quelque sorte une "subjectivité machinique " pour signifier que cette caractérisation est le produit d'une intelligence humaine. L'intelligence du concepteur doit être prise en compte dans la description du dispositif, car elle s'ajoute au profilage des processus. Mais au-delà de cette démarche de "programmation créative ", pour nous, un dispositif fait œuvre quand il est suffisamment complexe pour dissimuler son déterminisme - du point de vue perceptif du concepteur. Autrement dit, un dispositif est achevé quand il surprend son concepteur!

Le paradigme de la subjectivité machinique tente de rassembler et d'aborder des notions exigeant académiquement des méthodes différentes, ainsi que de rendre 
compte de l'entrelacement des structures et des organismes humains et non-humains, notamment dans un dispositif artistique, et de prendre compte de leur coexistence inextricable. La subjectivité machinique propose de dépasser l'opposition entre objectivité et subjectivité par l'intrication des processus informatiques et de la participation humaine.

Aussi, la subjectivité machinique permet de saisir d'un même mouvement une partie de la complexité de la création numérique qui est au cœur de l'élaboration du dispositif, en prenant en compte des facteurs objectifs (les senseurs, les traitements) appartenant à un cadre déterministe en même temps que des facteurs subjectifs (catégorisation, qualités) provenant du designer, du créateur, du participant, entre autres. Ce croisement de données ouvre sur un champ d'action interdisciplinaire.

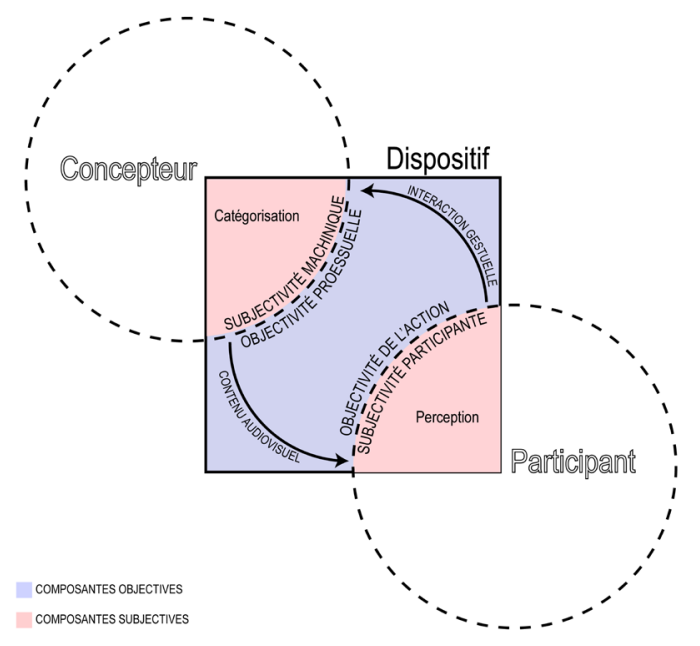

Figure 7: Les composantes objectives/subjectives du dispositif.

INTENTION COMPOSITIONNELLE ET INTERACTION GESTUELLE

Abordons un autre aspect de notre démarche de création. Nous l'avons vu, le geste est décrit selon trois modalités de reconnaissance de « soi » dans l'action, soit le geste physiologique, le geste musical direct et le geste de commande. Nous allons expliquer dans les lignes qui suivent l'articulation entre notre intention compositionnelle et l'interaction gestuelle.

Notre approche de la composition musicale liée au geste utilise la métaphore de la stratification de couche de son. Chaque couche correspond à un plan d'écoute-action, où un type d'interaction met en œuvre une séquence musicale. La composition de ces séquences forme l'œuvre. Prises séparément, les couches sonores sont chacune une situation singulière de reconnaissance de la forme musicale produite par un geste, à des niveaux structurels différents. À cela s'ajoute la complexité intrinsèque du contexte social des paysages sonores agissant sur l'interprétation de sa signification. Enfin, l'horizon sonore est composé pour plaire à l'écoute vagabonde, suivant la sonorité des timbres et leur facilité à être décelé à travers l'ambiance sonore.

Dans cette création, la composition met en équilibre des passages continus et homogènes : 
- le plan du paysage sonore que la cohérence dans la subjectivité de l'auditeur regroupe dans une « scène sonore » (Piché 2010, p. 111);

- le plan synthétique, aux accents de musique électronique, qui apporte une cohésion rythmique spatialisée qui lie l'ensemble ${ }^{19}$ et des "irruptions ", des accidents sonores qui rompent et provoquent l'expérience de la durée ;

- le plan des interventions ponctuelles. Le cycle de perception-action est mis en œuvre et entraîne l'écoute dans une " relation entre la continuité et la discontinuité »(Godøy 2009, p. 120). Suivant les surprises de l'écoute, les gestes physiologiques provoquent des ruptures, des accélérations de tempo qui à leur tour modifient l'ensemble du comportement du système.

Le plan du paysage sonore établit la couche de fond. Nous l'entreprenons en opposant une approche concrète à une approche plus sociale. D'une part, sous l'influence du projet de médiation, la captation devient un théâtre sonore durant lequel les jeunes performent leur rencontre à la musicalité des lieux, notre travail consistant alors à la documentation de ce moment. D'autre part, lors de l'édition et du montage sonore, le son est pris pour lui-même, détaché de sa signification, décontextualisé. Le son est compris pour son potentiel expressif par une écoute attentive de ses qualités concrètes et orientée par son devenir de matière dans laquelle des boucles répétitives seront découpées, jusqu'à en extraire le grain. Cependant, certaines traces du contexte social persistent à cause de leur caractère perforant qui permet d'attirer l'attention, de diriger l'écoute.

Dans la version de studio, le plan du paysage sonore investissait quatre contextes sociaux sur quatre pistes sonores aux réverbérations fort différentes. La première piste sonore (écouter), en référence aux travaux de Luc Ferrari ${ }^{20}$, fait entendre les talons des chaussures d'une femme dans un tunnel souterrain. La seconde (écouter), en référence au poème "Pour faire le portrait d'un oiseau " de Jacques Prévert, met en scène un oiseau dans une cage à barreaux de fer entouré par les enfants l'ayant sauvé. La troisième (écouter) est un emprunt au projet Rencontre dans les bois (2007), un paysage sonore forestier. Et, la quatrième piste (écouter), du noise ${ }^{21}$, représente le chaos et sa forte densité construit un effet « de mur » sonore. Le grand contraste entre ces pistes facilitait la compréhension de l'interaction. En effet, les participants percevaient facilement le changement sonore en rapport à leur mouvement de tête, particulièrement entre le noise et les claquements de talons. Tourner la tête pour entendre le noise semblait amplifier ce mouvement de manière presque cinématographique.

L'intention de mixité sonore joue sur deux tableaux : la mixité pour elle-même, pour sa densité d'une part, et d'autre part le mélange des ambiances et par conséquent le mélange des référentiels spatiaux. De manière exploratoire, la perception de l'espace

19 Extrait : https://soundcloud.com/jean-ambroise-vesac/chord-synthetique (consulté le 6 juin 2016).

20 Entrevue avec Christian Zanési dans l'émission Électrain de nuit du 15 décembre 2013 sur France Culture.

21 Les sources sonores sont produites par un Korg MS10, un Moog Minitaur, un Arturia Microbrute. 
et l'audition sont convoquées ensemble par le moment de l'écoute. Avec l'objectif de créer des moments d'écoute déroutants, ambigus, où se mêlent des scènes sonores de couloirs souterrains et des sons d'un parc extérieur. La mémoire du corps et des lieux est sollicitée par les références sonores. Évidemment, le participant peut prendre conscience de ses réactions physiologiques. À cette condition, il sera capable volontairement de déclencher des échantillons, par un geste devenu plus musical.

Le " geste musical direct" est un geste de type instrumental ${ }^{22}$. Dans les dispositions actuelles du système, les mouvements sont assez rudimentaires. Nous avons assigné l'orientation horizontale sur 360 degrés, divisés en quarts, au remix des pistes sonores. L'orientation cardinale est culturellement chargée de signification, propre à chaque culture et à chacun, pour cette raison, et en rapport aux quatre faces perpendiculaires au sol de l'espace cubique de l'installation. De manière simple, une piste sonore est assignée à chaque face du cube. Ainsi, le participant remixe, passe d'une piste à l'autre en tournant sur lui-même. Ce niveau d'interaction est simple, clair. La première chose que fait le participant est de vérifier comment et si l'installation fonctionne ; il cherche les indices de son action sur le système. Cette première étape est essentielle à l'expérience, car elle renvoie une image positive au participant. D'une certaine manière, l'installation lui signifie qu'il en a compris le fonctionnement. Sur cette base de confiance rudimentaire, le participant s'engage alors dans l'exploration. À partir de quelques points de repère, il laisse aller son imagination.

Le plan des interventions ponctuelles est un espace d'inscription de soi dans l'œuvre et en représente le niveau le plus ludique. Les gestes soudains de la tête, les mouvements brusques que l'on fait quand on change le regard de place sont détectés par la variation d'amplitude du mouvement dans un temps fixé. L'amplitude d'un geste brusque déclenche la lecture d'un « indice sonore ${ }^{23}$ » de quelques secondes qui se superpose au paysage sonore de fond. C'est le changement d'attitude qui est recherché, car il nous semble le mieux manifester un mouvement intérieur de la pensée. Trois niveaux sont utilisés pour les décrire : petits mouvements, mouvements moyens et grands mouvements. Les mouvements moyens ont été choisis pour déclencher une ponctuation sonore, intervenant sans quantification rythmique. L'échantillon sonore joué est spatialisé sur les huit haut-parleurs par une librairie ambiophonique ( $\operatorname{cosm} \sim)$. La spatialisation est elle-même contrôlée par l'orientation et suit les mouvements actuels de la tête du participant. Ce contrôle direct est voulu pour renforcer le plaisir du jeu sonore.

Par ces mouvements, le participant actualise le montage sonore qui est pour nous " une forme minimale d'écriture " (Kaltenecker 2013, p. 527). Le temps s'organise à partir d'événements dans la perception du participant, appliqués au programme informatique. Cependant, dans une installation interactive prévue pour des non-mu-

22 Nous ne nous attarderons pas sur la notion qui est en dehors de notre objet d'étude.

23 "L'indice sonore matérialisant désigne un aspect d'un son, quel qu'il soit, qui fait ressentir plus ou moins précisément la nature matérielle de sa source et l'histoire concrète de son émission » (Chion 2010, p. 110). 
siciens, l'exécution ne peut être uniquement déterminée par l'interaction, car les participants n'ont pas tous l'élan pour entrer en musique. Pour y remédier, les pistes sonores de base servent de courroie d'entraînement.

Le choix de l'échantillon joué est déterminé par l'indice de l'état du comportement de l'auditeur qui interagit dans l'installation, tel que défini par la formalisation des données des senseurs. Le geste de commande est l'acte que pose le participant en cherchant à provoquer une réaction de haut niveau du système. Cette catégorisation comprend cinq niveaux pour correspondre aux comportements : absent, calme, normal, agité et fou. La progression de l'intensité comportementale est traduite dans le son par l'intensification de la présence du contexte social dans le paysage sonore, pouvant passer d'un paysage sonore hivernal capté sur les rives du Saint-Laurent à des rires captés lors des ateliers de médiation avec des groupes de jeune du quartier ${ }^{24}$, en passant par les bruits des rues de Taïwan ${ }^{25}$. La voix - celle de la soprano Peggy Bélanger à l'intérieur de l'église de Saint-Jean-Port-Joli (Québec) - est particulièrement facile à localiser et est préférablement utilisée dans cette couche fortement spatialisée. La musicalité des échantillons est de plus en plus prononcée. La musicalité des paysages sonores est mise au jour par la répétitivité des extraits lus en boucle et dont les rythmes s'entrechoquent. Ici, la musicalité est définie par une suite de bruits aux timbres distincts, semblant se répondre par un effet de cohérence d'ensemble. Cette cohérence est en partie ressentie par l'effet de corporalité des mouvements sonores et leur évocation « visuelle ». De ce fait, les échantillons sont sélectionnés pour leur potentiel à évoquer un corps en mouvement, une action, une situation, un lieu, une ambiance où l'imagination se plonge : une immersion incorporante. $\mathrm{Au}$ dernier degré d'écoute, le son est devenu un bruit hérissé et brut. Le " grain du son » est la dernière échelle avant le rien sonore, car il n'y a plus aucune trace du son matériel d'origine. L'écoute est submergée par une matière hypnotique qui révèle les artéfacts électroniques de la diffusion et son effet psychédélique.

Enfin, le plan synthétique assoit la musicalité de l'installation à la manière d'un chœur de voix synthétiques dont les harmonies soutiennent l'écoute ; il évoque le jeu sensible du moment. Les timbres ronds sont utilisés pour ne pas trop adresser l'attention et ainsi laisser de la place pour les sons brefs plus définis de la couche d'intervention ponctuelle spatialisée.

\section{MÉDiATiON CULTURELLE}

À présent, changeons de registre et abordons une autre perspective de notre démarche de création : la médiation culturelle. Assez récemment, la médiation est devenue incontournable dans le milieu culturel. Elle s'est imposée par la volonté

24 Vidéo disponible en ligne : http://youtu.be/HAYrXrgixlA (consulté le 6 juin 2016). Cette démarche a été inspirée de Pierre Redon et son projet Tülü. Voir : http://marchesonore.com/my-product/tulu/ (consulté le 6 juin 2016).

25 Montage sonore disponible en ligne : https://soundcloud.com/jean-ambroise-vesac/soleil-mediation-audio (consulté le 6 juin 2016). 
politique. Les objectifs de la médiation culturelle sont multiples ${ }^{26}$ : le développement de nouveau public, l'accessibilité de l'offre culturelle à des groupes sociaux défavorisés, l'encouragement de la fréquentation des espaces culturels et par extension des institutions.

Les approches de la médiation sont nombreuses. Selon nous, l'approche pratique de " main à la patte " est la plus à même de réussir. L'attention du participant sera grande si une partie de son travail est présentée publiquement. Le projet de médiation de Soleil reprend donc une sélection des meilleurs résultats d'ateliers en guise de contenu sonore de l'œuvre. De plus, en préparation à une visite de l'installation, un second volet de l'atelier de médiation sert de premier contact des participants avec l'interface interactive. Préalablement, il faut introduire le participant à ce qui semble être pour lui " un nouveau médium ", par une conscientisation perceptive. En effet, dans le cas d'une interface d'interaction encore inconnue, le participant doit pouvoir associer ses mouvements à des réponses sensibles. Il forme ainsi un nouvel arc de perception-action dans son "schéma sonore ». C'est pourquoi les participants testent les lunettes et l'interactivité avec une version simplifiée du programme. À la fin de cet atelier, les participants sont doublement préparés pour une visite en galerie d'art : d'une part, ils savent globalement comme celle-ci fonctionne, et, d'autre part, ils sont fiers de retrouver une partie de leur contribution dans l'œuvre exposée.

En résumé, l'œuvre Soleil intègre son projet de médiation culturelle et artistique au sein de ses processus et de ses contenus. Les effets bénéfiques de la valorisation de la culture et d'une approche sensible du monde sont nombreux, aussi bien pour l'estime de soi que pour l'ouverture à l'autre, ainsi que pour l'affermissement du sentiment d'appartenance à la communauté, au territoire, à la société. Pris dans un sens littéral, le sentiment d'appartenance se rapporte à la situation du sujet dans son milieu, entraînant l'impression " d'être à sa place ». "Être bien ici » est une qualité subjective de la relation $\mathrm{du}$ " corps vécu » et du monde : "Dans les processus cognitifs tissant nos relations à l'environnement le système vestibulaire joue un rôle central » (Berthoz et Christen 2009, p. 21). Au premier plan, 1'audition joue un rôle majeur dans notre rapport au monde par sa proximité physique et cognitive dans la reconstruction de la cohérence spatiale des relations du corps et de l'espace (Berthoz 2010, p. 343) et par son rôle dans l'orientation sonore.

Ainsi donc, nous soulignons le lien entre le sentiment "d'être à sa place dans le monde " et la relation du corps à son espace, particulièrement dans l'expérience du paysage sonore. Dans l'hypothèse où notre participation au monde serait relative, en partie, à notre rapport à son espace sonore, un paysage sonore peut-il être un espace de médiation socioculturelle par l'instrumentalisation de l'écoute ? La musicalité permet-elle de réfléchir notre participation au monde?

Sans être narratif, le paysage sonore offre des ambiances qui peuvent faire voyager, qui rappellent des souvenirs, qui restituent des situations sociales. L'évolution de ces

26 Des exemples sont disponibles sur le site de la Ville de Montréal, en ligne : http://montreal.mediationculturelle.org (consulté le 6 juin 2016). 
situations, l'enchaînement des évènements sonores sont des points d'écoute indiciels. C'est une captation du réel presque documentaire et non une fiction. Les jeunes qui participent à l'activité de médiation ${ }^{27}$ sont conscients d'être enregistrés, ce qui les " met en scène ». Ils jouent à partir des matériaux et des éléments de leur environnement direct. En plus de point d'écoute, les traces des gestes conscients cherchant une musicalité constituent des empruntes, des indices d'une narration, des témoins de la vie. L'exploration de la musicalité du monde, dans une démarche d'écoute " très " active, ajoute à l'expérience des participants de leur territoire une interprétation déviante de leur quotidien, utile à la réappropriation " enchantée » du paysage de leur vie.

Le projet de médiation a fourni trois paysages sonores urbains de trois quartiers différents de Montréal-Nord. Rappelons que l'intention de création était de permettre à l'imagination de voyager entre ces espaces. Malheureusement, les matériaux sonores capturés lors des ateliers de médiation ont rendu la reconnaissance des différentes pistes difficile, à cause de leur trop grande ressemblance sonore liée à leur proximité contextuelle. Une majorité de participants, n'ayant pas d'habitude de la création musicale ou de l'écoute spatialisée, ne pouvait plus faire la différence entre les parties. Ainsi donc, c'est un pan complet de l'interaction qui devenait imperceptible. Ce constat révèle peut-être moins la faiblesse des enregistrements que le manque de repères sonores distinctifs et constitutifs de ces espaces urbains.

Selon nous, la médiation participe au cœur de l'œuvre. Pour cette raison, cette contrainte de création doit être adressée le plus tôt possible dans le cycle du projet. En effet, il ne s'agit pas de "donner une manette de télécommande » au participant lui permettant de "zapper », mais bien de dépasser la notion d'interface pour tenter d'accéder à une relation symbiotique des agents humains et non-humains du dispositif. À cet effet, le participant est pris comme point de référence ("point de fitness ») pour la partie informatique du système, les processus informatiques s'adaptent à lui. Ainsi, $\mathrm{du}$ fait de son emprise sur les processus, parfois sur les objectifs, le participant est incorporé à l'ensemble technique constituant le dispositif. Il imprime sa sensibilité dans l'œuvre.

\section{RÉCEPTION}

Du versant del'immersion, la reconnaissance de soi dans les processus de commande audiovisuelle de haut niveau, mène à une implication incorporante à l'œuvre. À ce point, la participation est une incorporation immersive. En outre, par ses actions, son intention d'être-là et son attention portée sur le contenu du dispositif, le participant

27 Grâce au soutien financier de la Ville de Montréal, des activités de médiation se sont déroulées en lien avec l'exposition de l'installation à la Maison culturelle Montréal-Nord. Les jeunes ont eu une occasion unique de participer à la construction de paysages sonores reliés à l'œuvre. Trois équipes ont été rencontrées pour réaliser cette création. Une incursion dans divers lieux à proximité de la Maison culturelle a permis aux jeunes non seulement de capter des sons et des bruits ambiants, mais aussi d'en générer. La partition musicale ainsi constituée a été par la suite intégrée à l'œuvre. La vidéo est disponible en ligne : http://youtu.be/r2n1Km2D0UQ (consulté le 6 juin 2016). 
expérimente une incorporation projective, parce qu'il fait une simulation mentale des états futurs possibles du dispositif.

L'observation des ateliers de médiation et des réactions des participants nous informe de la diversité des "personnalités ». Les participants rentrant dans le dispositif, suite à une petite attente dans la salle d'exposition, sont rarement seuls. Ils arrivent en petit groupe d'amis, et un après l'autre se lancent dans l'expérience. De ce que nous avons pu voir, et sans plus d'étude, les mouvements du corps sont la plupart du temps assez dansant. Les jeunes, particulièrement, bougent comme ils le feraient sur une piste de danse. Il nous semble que beaucoup d'entre eux ne savent que faire. Ils rentrent et qu'attendent-ils, un buzz ? Il ne vient pas immédiatement à l'idée d'écouter, de chercher le retour de force du système dans les sons et dans l'organisation musicale. Cette attitude provient peut-être de la nouveauté de la proposition et du peu de référents culturels permettant d'imaginer ce à quoi l'art de l'installation amène à sentir. Cette installation et la médiation offrent une occasion de vivre un être-là dans l'art, à l'art.

Certaines personnes ont des habiletés d'analyse de leur environnement stupéfiantes. Ces individus que l'on pourrait appeler hypersensibles sont aussi hyperconscients de ce qui se passe. Ils sont en mesure d'apprécier la synchronisation son-lumière. La précision de celle-ci se situe dans le cadre du décalage de vitesse de traitement électronique de la diffusion sonore (latence) et la latence du circuit de lumière DEL entre 15 et $50 \mathrm{~ms}$.

D'autres personnes sont plus sensibles au niveau des intentions artistiques et comprennent ce à quoi la composition sonore conduit, en termes de qualité musicale et d'implication sociale. Il semble que ces personnes analysent les segments et les couches de la composition pour les associer à leur contexte d'origine. Le mélange de ces différentes provenances est facilement accepté pour des oreilles ouvertes à des styles musicaux utilisant l'échantillonnage sonore, comme le hip-hop.

Enfin, des amateurs d'art vivent l'expérience comme une entrée dans la couleur et le sensible. L'installation est alors acceptée comme un signe de forme future d'œuvres qui seront des véhicules extraordinaires, une forme insensée de surréalisme/fiction. À ce point de projection, les plus jeunes les rejoignent dans l'expectative de « la machine à voyager dans le temps ». Il y a donc des attentes irrationnelles parcourant la salle d'exposition, une fantaisie s'abreuvant à une pensée magique, une superstitieuse persistance envers les technologies.

$$
* * *
$$

En conclusion, pour rompre avec la prévisibilité des dispositifs d'interaction corporelle simple, l'intégration du participant au cœur des processus de l'œuvre présente la variabilité potentiellement imprédictible requise à l'évolution de l'œuvre. L'interaction se situant bien au niveau des actes de commandes, et non pas uniquement à un niveau instrumental, conserve son accessibilité et la trace de son agentivité d'origine.

Le dispositif Soleil forme un ensemble complexe aux propriétés émergentes. L'exploration de ces possibilités d'émergence requiert une conception intégrée des 
traitements informatiques, des contenus médiatiques et de l'implication sensible. Le paradigme de la subjectivité machinique permet une telle démarche de création.

Mais, quand la frontière entre humain et non-humain se dilate et devient poreuse, une autre limite se redéfinit. Et si ce n'était pas moi ? Quelle est la part qui revient au " créateur » dans une œuvre qui engage la participation et qui transfert une grande partie de son organisation à des processus machiniques? Est-ce un dépassement ou une perte de soi?

\section{BiBLIOGRAPHIE}

Agamben, Giorgio (2007), Qu'est-ce qu'un dispositif ?, Paris, Payot \& Rivages.

Alberganti, Alain (2013), De l'art de l'installation. La spatialité immersive, Paris, L'Harmattan.

Berthoz, Alain (2010), «Physiologie de la perception et de l'action », Annuaire du Collège de France 20082009, résumé des cours et travaux $109^{\mathrm{e}}$ année, p. 371-403, https://annuaire-cdf.revues.org/358, consulté le 6 juin 2016.

Berthoz, Alain, et Yves Christen (dir.) (2009), Neurobiology of "Umwelt". How Living Beings Perceive the World, Berlin, Springer.

Bioteau, Alain (2013), "Spatialisation et écriture de l'espace, des pionniers à Emmanuel Nunes ", dans Nicolas Donin et Laurent Feneyrou (dir.), Théories de la composition musicale au XX siècle, vol. 2, Lyon, Symétrie, p. 1337-1366.

Boisclair, Louise (2013), "Exuvie chorégraphique de Choinière-Ballard et Migration numérique de Vesac. Personnage virtuel, figure ou avatar? ", dans Renée Bourassa et Louise Poissant (dir.), Personnage virtuel et corps performatif. Effets de présence, Québec, Presses de l'Université du Québec, p. 149-168.

Bonenfant, Maude (2013), "Rôle de l'avatar et identité en ligne dans les jeux vidéo », dans Renée Bourassa et Louise Poissant (dir.), Avatars, personnages et acteurs virtuels, Québec, Presses de l'Université du Québec, p. 171-193.

Bosseur, Jean-Yves (2013), « L'école de New York », dans Nicolas Donin et Laurent Feneyrou (dir.), Théories de la composition musicale au XX' siècle, vol. 1, Lyon, Symétrie, p. 623-638.

Botte, Marie-Claire, et al. (1989), Psychoacoustique et perception auditive, Paris, INSERM.

Cage, John, et David Tudor (1959), Indeterminacy. New Aspect of Form in Instrumental and Electronic Music, New York, FT 3704 Folkways.

Camurri, Antonio (2000), «Artificial Intelligence Architectures for Composition and Performance Environment ", dans Eduardo Reck Miranda (dir.), Readings in Music and Artificial Intelligence, Amsterdam, Harwood Academic, p. 163-188.

Carman, Taylor (1999), "The Body in Husserl and Merleau-Ponty », Philosophical Topics, vol. 27, $\mathrm{n}^{\circ}$ 2, p. 205-226, http://ist-socrates.berkeley.edu/ hdreyfus/188 s05/pdf/Carman Body.pdf, consulté le 29 juin 2016.

Chion, Michel (2010), Le son. Traité d'acoulogie, $2^{\mathrm{e}}$ éd. rev. et corr., Paris, Armand Colin.

Chion, Michel (2013), L'audio-vision. Son et image au cinéma, $3^{e}$ éd., Paris, Armand Colin.

Couchot, Edmond, et Norbert Hillaire (2003), L'art numérique, Paris, Flammarion.

Craighero, Laila, et al. (1999), "Action for Perception. A Motor-visual Attention Effect ", Journal of Experimental Psychology. Human Perception and Performance, vol. 25, $\mathrm{n}^{\circ}$ 6, p. 1673-1692, http://web.unife.it/progetti/neurolab/pdf/1999 3 Craighero-Fadiga Action\%20for\%20

Perception.pdf, consulté le 6 juin 2016. 
Dubé, Francis (2013), « Préface », dans Sophie Stévance et Serge Lacasse (dir.), Les enjeux de la recherchecréation en musique, Québec, Presses de l'Université Laval, p. xi-xvi, http://livres.prologuenumerique. ca/Telechargement/Extrait.cfm?ISBN=9782763719511\&type=pdf, consulté le 6 juin 2016.

Falcão, Thiago (2012), "Structures of Agency in Virtual Worlds. Fictional Worlds and the Shaping of an In-Game Social Conduct », dans Nelson Zagalo, Leonel Morgado et Ana Boa-Ventura (dir.), Virtual Worlds and Metaverse Platforms. New Communication and Identity Paradigms, Hershey, PA, Information Science Reference, p. 192-205, www.academia.edu/2482976/Structures of Agency in Virtual Worlds Fictional Worlds and the Shaping of an In-Game Social Conduct, consulté le 29 juin 2016.

Farnell, Andy (2010), Designing Sound, Cambridge, Mass., MIT Press.

Fourneret, Pierre (2002), « De la perception de l'action à la conscience de Soi. Contribution de la neuropsychologie cognitive aux problèmes de la reconnaissance et de l'identification du corps agissant ", dans Yann Coello et Jacques Honoré (dir.), Percevoir, s'orienter et agir dans l'espace. Approche pluridisciplinaire des relations perception-action, Marseille, Solal, p. 251-266.

Gangopadhyay, Nivedita, Madary, Michael, et Finn Spicer (dir.) (2010), Perception, Action, and Consciousness. Sensorimotor Dynamics and Two Visual Systems, Oxford, Oxford University Press.

Gell, Alfred (2009), L'art et ses agents, Les presses du réel, Dijon.

Godøy, Rolf I. (2009), "Gesture Affordances of Musical Sound », dans Rolf I. Godøy et Marc Leman (dir.), Musical Gestures. Sound, Movement, and Meaning, New York, Routledge, p. 103-125.

Grau, Oliver (2003), Virtual Art. From Illusion to Immersion, Cambridge, Mass., MIT Press.

Hansen, Mark B. N. (2006), New Philosophy for New Media, Cambridge, Mass., MIT Press.

Jankélévitvh, Vladinir (1983), La musique et l'ineffable, Paris, Seuil.

Jeannerod, Marc (2006), Motor Cognition. What Actions Tell the Self, Oxford, Oxford University Press.

Jones, Caroline A. (2006), Sensorium, Embodied Experience, Technology, and Contemporary Art, Cambridge, Mass., The MIT Press.

Kaltenecker, Martin (2013), "De l'art radiophonique au paysage sonore », dans Nicolas Donin et Laurent Feneyrou (dir.), Théories de la composition musicale au XX'e siècle, vol. 1, Lyon, Symétrie, p. $507-538$.

Liestøl, Gunnar (2009), «Augmented Reality and Digital Genre Design-Situated Simulations on the iPhone ", 2009 IEE International Symposium on Mixed and Augmented Reality-Arts, Media and Humanities (29-22 octobre), p. 29-34, http:/ /ieexplore.ieee.org/xpl/mostRecentIssue. jsp?punumber $=5319228$, consulté le 6 juin 2016.

Lussac, Olivier (2013), "Dadaïsme, surréalisme, futurisme... et Fluxus », dans Nicolas Donin et Laurent Feneyrou (dir.), Théories de la composition musicale au XXe siècle, vol. 1, Lyon, Symétrie, p. $541-575$.

Maciunas, George (1990), « Neo-Dada in Music, Theater, Poetry, Art », lu par Arthur C. Caspari au concert Fluxus « Après John Cage » (9 juin 1962), Wuppertal, Allemagne, dans Gino Di Maggio (dir.), Ubi Fluxus ibi Motus, 1990-1962, Milan, Mazzotta, p. 214-225, www.kim-cohen.com/seth texts/artmusictheorytexts/Maciunas Neo\%20Dada.pdf, consulté le 6 juin 2016.

Mariétan, Pierre (2005), Écrits de musique, «Vol. 2. L'environnement sonore. Approche sensible, concepts, modes de représentation », Nîmes, Champ social.

Nicolas, Serge, et Karine Doré-Mazars (2002), «Éléments d'histoire sur la perception et les liens perception-action », dans Yann Coello et Jacques Honoré (dir.), Percevoir, s'orienter et agir dans l'espace. Approche pluridisciplinaire des relations perception-action, Marseille, Solal, p. 15-29.

Piché, Jean (2010), L'odyssée des médias-son. Fondements théoriques et pratiques de l'approche ambiophone de l'environnement sonore et de la scène auditive à validité écologique, Saarbruck, Éditions universitaires européennes.

Pickering, Andrew (1995), The Mangle of Practice. Time, Agency and Science, Chicago, University of Chicago Press. 
Raffnsøe, Sverre (2008), "Qu'est-ce qu'un dispositif ? L'analytique sociale de Michel Foucault », Symposium (Canadian Journal of Continental Philosophy/Revue canadienne de philosophie continentale), vol. 12, no 1, Article 5, p. 44-66, http://xn--raffnse-vla.com/?p=1000, consulté le 6 juin 2016.

Renaut, Alain (1988), "Les subjectivités. Pour une histoire du concept de sujet ", dans Elisabeth Guibert-Sledziewski et Jean-Louis Vieillard-Baron (dir.), Penser le sujet aujourd'hui, Paris, Méridiens Klincksieck, p. 55-77.

Richard, François, et Steven Wainrib (dir.) (2006), La subjectivation, Paris, Dunod.

Russolo, Luigi (1975), L'art des bruits, textes établis et présentés par Giovanni Lista, traduits par Nina Sparta, Lausanne, L'âge d'homme.

Sabbe, Herman (2013), " György Ligeti ", dans Nicolas Donin et Laurent Feneyrou (dir.), Théories de la composition musicale au XX siècle, vol. 2, Lyon, Symétrie, p. 1081-1094.

Schellenberg, Susanna (2011), " Perceptual Experience and the Capacity to Act ", dans Nivedita Gangopadhyay, Michael Madary et Finn Spicer (dir.), Perception, Action, and Consciousness. Sensorimotor Dynamics and Two Visual Systems, Oxford, Oxford University Press, p. 145-159, http://philpapers.org/rec/SCHSAT-9, consulté le 6 juin 2016.

Schöne, Hermann (1984), Spatial Orientation. The Spatial Control of Behavior in Animals and Man, Princeton, N.J., Princeton University Press.

Wiener, Norbert ([1954]1988), The Human Use of Human Beings. Cybernetics and Society, New York, Da Capo Press. 